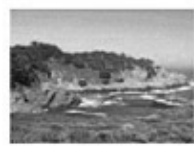

Western Medical Research conference (formerly Western Regional Meeting) January 24-26 2019 Carmel, California
Western Section American Federation for Medical Research

Western Society for Clinical Investigation

Western Association of Physicians

Western Society for Pediatric Research

Western Student and Resident Medical Research Forum

California Thoracic Society

\title{
WESTERN MEDICAL RESEARCH CONFERENCE (FORMERLY WESTERN REGIONAL MEETING)
}

\section{ABSTRACTS \& AWARD WINNERS}

Ahmed Alaini, University of New Mexico

Karissa Aldrete, University of New Mexico Health Sciences Center Shehabaldin Alqalyoobi, University of California, Davis

Lauren Au, Hawaii Pacific Health

Jorge Avila, University of California, Davis

Clare Batty, University of New Mexico

Dylan Bergstedt, Colorado University Anschutz Medical Campus

Joseph Bircher, University of Utah

Gabriel Blank, University of British Columbia

Ethan Bodle, Stanford University

Andrew Bosomworth, Western University of Health Sciences

Marcus Brisco, John A Burns School of Medicine

Noemi Capistrano, Harbor-UCLA Medical Center

Rachael Carricaburu, University of Washington

Lauren Carter, Stanford University

Stephanie Chassen, University of Colorado

Alison Chu, David Geffen School of Medicine at UCLA

Sarah Cilvik, University of Colorado School of Medicine

Sydney Cooper, University of New Mexico

Shanlee Davis, University of Colorado

Mitchell Dumais, University of Washington

Rebecca Dutta, University of California, Davis

Viviana Fajardo, University of California Los Angeles

Imaneh Fallahi, University of New Mexico Health Sciences Center

Manuel Flores, Charles R. Drew University

Babalola Funmilola, Loma Linda University

Balram Gangaram, University of California San Francisco

Kimberley Gerard, University of California, Davis

Gregory Goldstein, Stanford University

Cynthia Gong, Children's Hospital Los Angeles

Thoa Ha, University of California San Francisco

Kaidi He, University of California Irvine

Sara Heard, University of Colorado School of Medicine

Danielle Jacobsen, Stanford University School of Medicine

Pratichi Kadam, British Columbia Children's Hospital

Vaneet Kalra, UC Davis Children's Hospital

Sheila Kalyanam, Harbor-UCLA

Jenny Koo, University of California San Diego

Serena Laing, Shriner's Hospitals for Children Northern California Amy Lebedoff, University of Utah

Shu-Yi Liao, University of California, Davis

Jay Martinez, University of New Mexico Health Sciences Center

Antonie Meixel, University of California San Diego

Jacob Michalski, University of Colorado

Shannon Mumenthaler, University of Southern California

Neeharika Namineni, University of California San Diego

David Nathan, Charles R. Drew University of Medicine and Science

Ananya Nrusimha, Stanford University

Urvashi Parti, University of Southern California

Alyssa Perez, University of California San Francisco

Jennifer Rosenbaum, University of Washington

Eniko Sajti, University of California, San Diego

Laura Sherlock, University of Colorado

Tania Torbati, Cedars-Sinai Medical Center

Dara Torgerson, McGill University

Kristen Wigby, University of California, San Diego

Amy Yeh, LAC+USC Keck School of Medicine of USC

Brooke Zhao, University of Utah
WAP/WSCI Travel Award Winner

WAFMR/WAP/WSCI Student Subspecialty Award Winner

California Thoracic Society Scholar Winner

WSPR Mead Johnson Travel Award Winner

WAFMR/WAP/WSCI Student Subspecialty Award Winner

WSPR Abbott Nutrition Lowell Glasgow Student Research Award Winner WAFMR/WAP/WSCI Student Subspecialty Award Winner

WAP/WSCI Travel Award Winner

WAFMR/WAP/WSCI Student Subspecialty Award Winner

WSPR Abbott Nutrition Subspecialty Award

WAFMR/WAP Student Subspecialty Award Winner

WSPR Abbott Nutrition Subspecialty Award

AFMR Henry Christian Awardee

WAFMR/WAP/WSCI Student Subspecialty Award Winner

AFMR Henry Christian Awardee

WSPR Mead Johnson Nutrition Junior Faculty Travel Award

WSPR Mead Johnson Nutrition Junior Faculty Travel Award

AFMR Henry Christian Awardee

WSPR Abbott Nutrition Lowell Glasgow Student Research Award Winner Mallinckrodt Junior Faculty Travel Award - Neonatal Pulmonary

WAFMR/WAP/WSCI Student Subspecialty Award Winner

WSPR Abbott Nutrition Subspecialty Award

WAP/WSCI Travel Award Winner

WAFMR/WAP/WSCI Outstanding Resident Research Award Winner

WAFMR/WAP/WSCI Student Subspecialty Award Winner

WAP/WSCI Travel Award Winner

WSPR Mead Johnson Travel Award Winner

Inaugural Vedang Londhe Memorial Travel Award

AFMR Henry Christian Awardee

WAP/WSCI Travel Award Winner

WSPR Abbott Nutrition Subspecialty Award

WSPR Outstanding Resident Research Award Winner

WSPR Mead Johnson Travel Award Winner

WSPR Abbott Nutrition Subspecialty Award

WAP/WSCI Travel Award Winner

WSPR Mead Johnson Nutrition Junior Faculty Travel Award

WSPR Abbott Nutrition Subspecialty Award

WSPR Mead Johnson Travel Award Winner

WAP/WSCI Travel Award Winner

WSPR Mead Johnson Travel Award Winner

AFMR Henry Christian Awardee

WAFMR/WAP/WSCI Student Subspecialty Award Winner

WSPR Abbott Nutrition Subspecialty Award

Edwin E. Osgood Award Winner

WAFMR Outstanding Investigator Award Winner

WAFMR/WAP/WSCI Student Subspecialty Award Winner

WAFMR/WAP/WSCI Student Subspecialty Award Winner

WSPR Mead Johnson Travel Award Winner

WAFMR/WAP/WSCI Student Subspecialty Award Winner

AFMR Henry Christian Awardee

WAP/WSCI Travel Award Winner

Mallinckrodt Junior Faculty Travel Award - Neonatal Pulmonary

WAP/WSCI Travel Award Winner

WAFMR/WAP/WSCI Student Subspecialty Award Winner

WSPR Mead Johnson Nutrition Junior Faculty Travel Award

WSPR Mead Johnson Nutrition Junior Faculty Travel Award

WSPR Abbott Nutrition David W. Smith Pediatric Trainee Research Award WSPR Mead Johnson Travel Award Winner 